\title{
Fatores de risco para óbito em Unidade de Terapia Intensiva Neonatal
}

\author{
Risk factors for death in the Neonatal Intensive Care Unit
}

Luiz Fernando C. Nascimento ${ }^{1}$

\section{RESUMO}

Objetivo: Estimar fatores de risco para óbito durante internação em uma Unidade de Terapia Intensiva Neonatal (UTIN) por modelo logístico hierarquizado.

Métodos: Trata-se de estudo observacional, analítico e longitudinal com recém-nascidos internados na UTIN de um hospital universitário, no período de janeiro/2000 a dezembro/2003. A variável dependente foi óbito intrahospitalar e as independentes foram variáveis antenatais, perinatais e pós-natais. Criou-se um modelo hierarquizado em três níveis. Realizada a análise bivariada, foram incluídas no modelo as que apresentavam $p<0,20$ e mantidas se $p<0,05$. O procedimento utilizou o programa SPSS 10.0 para a análise e estimativa da acurácia, adotando-se nível de significância de $5 \%$.

Resultados: Foram incluídos no estudo 367 recém-nascidos, tendo ocorrido 69 (18,8\%) óbitos no período. As variáveis com significância estatística que compuseram o modelo final foram: relato de natimorto anterior, ordem de nascimento, Apgar de quinto minuto inferior a 7 , idade gestacional inferior a 37 semanas e ventilação mecânica durante a internação. $\mathrm{O}$ modelo apresentou acurácia de 86,9\%.

Conclusões: $\mathrm{O}$ modelo obtido neste estudo contém variáveis dos três níveis hierárquicos e poderá ser utilizado em Unidades de Terapia Intensiva Neonatal que apresentem comportamento semelhante à unidade na qual se realizou este estudo.

Palavras-chave: prematuro; Unidade de Terapia Intensiva; mortalidade intra-hospitalar; fatores de risco; modelo logístico.

\section{ABSTRACT}

Objective: To estimate the risk factors for mortality during hospitalization in a Neonatal Intensive Care Unit (NICU) using a hierarchical logistic model.

Methods: This longitudinal, observational and analytical study enrolled newborns admitted to the NICU from January/2001 to December/2003. The outcome analyzed was in-hospital death and the independent variables were prenatal, perinatal and postnatal factors. A hierarchical model with three levels was built. The variables with $p<0.20$ by bivariated analysis were included in the model and, after adjustment at the same level, variables with $p<0.05$ were maintained in the logistic model. Statistic analysis was performed by SPSS.10, software that estimated the accuracy of the model, adopting significance as $p<0.05$.

Results: Among 367 newborns included, 69 (18.8\%) died during hospital stay. The following risk factors comprised the hierarchical model of logistic regression and were significantly associated with death among the studied neonates: previous stillbirth, being first or second child, Apgar at five minutes below 7, preterm newborn and use of mechanical ventilation. This model had $86.9 \%$ of accuracy.

Conclusions: The model obtained in this study has variables of the three hierarchical levels and might be used in Neonatal Intensive Care Units that share the same characteristic of the unit herein studied.

Key-words: infant, premature; Intensive Care Units; hospital mortality; risk factors; logistic model.
Instituição: Universidade de Taubaté (Unitau), Taubaté, São Paulo, Brasil ${ }^{1}$ Professor-assistente do Departamento de Medicina da Unitau, Taubaté, SP, Brasil; Doutor em Saúde Pública pela Universidade de São Paulo (USP), São Paulo, SP, Brasil
Endereço para correspondência:

Luiz Fernando C. Nascimento

Rua Durval Rocha, 500 - Vila Paraíba

CEP 12515-710 - Guaratinguetá/SP

E-mail: Ifcn@unitau.br

Recebido em: 21/7/08

Aceito em: 11/11/08 


\section{Introdução}

Baixo peso ao nascer, prematuridade, asfixia neonatal, infecções congênitas ou hospitalares, além de malformações não compatíveis com a vida, são reconhecidamente fatores de risco para o óbito infantil e, em especial, no período neonatal ${ }^{(1-3)}$. Esses dados geralmente são obtidos por meio de fontes oficiais, como o Sistema de Informações sobre Nascidos Vivos (Sinasc) do Ministério da Saúde e Sistema de Informações sobre Mortalidade (SIM). Os estudos utilizam técnicas como a regressão logística ou a regressão de $\operatorname{Cox}^{(4-6)}$ para identificar possíveis fatores de risco para o óbito neonatal.

Com o advento das Unidades de Terapia Intensiva Neonatal (UTIN), houve uma mudança considerável no prognóstico de recém-nascidos gravemente enfermos, criando-se escores preditivos para estimar a probabilidade ou possibilidade de óbito nesse grupo de crianças. Assim, foram criados o PRISM (Pediatric Risk of Mortality Score), SNAPPE (Score for Neonatal Acute Physiology-Perinatal Extension) e o CRIB (Clinical Risk Index for Babies) ${ }^{(7-9)}$, além de um modelo pouco convencional que utiliza a lógica $f u z z y^{(10)}$.

Estudos sobre as taxas de mortalidade em UTIN ainda são escassos no Brasil e, em geral, enfocam o componente neonatal e mostram proporções de óbitos que oscilam entre 10 e $48 \%$ das internações, segundo estudo realizado em Goiânia, em nove unidades de terapia intensiva neonatal ${ }^{(5)}$. Anteriormente, Zullini et al ${ }^{(11)}$ apontaram porcentagens de até $12 \%$ de óbitos em recém-nascidos internados em UTIN. Estudos que utilizaram o escore CRIB como preditor de mortalidade revelaram diferentes valores para a mortalidade: 21,23 e $30 \%^{(12-14)}$.

Determinantes distais de natureza socioeconômica e demográfica que ocorrem no período pré-natal e fatores perinatais, tais como peso ao nascer, idade gestacional e escore de Apgar, são apontados como preditores do óbito em UTIN ${ }^{(2-6)}$. Determinantes pós-natais representados pelos procedimentos invasivos, frequentes durante a internação em UTI, como o cateterismo de vasos umbilicais e a ventilação mecânica, bem como medidas curativas como o uso de surfactante e a exsanguíneotransfusão, além de intercorrências clínicas como a apneia e a convulsão também contribuem para o aumento do risco de óbito.

O objetivo deste estudo é descrever um modelo com fatores de risco para óbito nas UTIN, utilizando-se a técnica de regressão logística não condicional hierarquizada e incluindo-se eventos relativos à internação.

\section{Métodos}

Trata-se de estudo observacional, longitudinal e analítico, envolvendo recém-nascidos internados na UTIN do Hospital Universitário de Taubaté, SP, no período correspondente aos anos de 2000 a 2003. Foram excluídos os recém-nascidos transferidos de outros municípios para a UTIN e os que evoluíram a óbito nas primeiras 12 horas de internação. Este estudo foi aprovado pelo Comitê de Ética em Pesquisa da Universidade de Taubaté.

Taubaté situa-se no cone leste paulista, mais especificamente no Vale do Paraíba, a cerca de $120 \mathrm{~km}$ de São Paulo, com uma população de aproximadamente 250 mil habitantes. É referência no atendimento hospitalar e conta com um hospital universitário. Atende pacientes do próprio hospital universitário predominantemente e pacientes transferidos de outros municípios da região. No período de realização deste estudo, a UTIN tinha capacidade para assistir seis recém-nascidos.

A variável dependente considerada foi o óbito durante a internação, não se levando em consideração a data do evento, isto é, se ocorrido no período neonatal ou pós-neonatal. As variáveis independentes foram classificadas em pré-natais, perinatais e pós-natais, sendo que esta última continha informações sobre a evolução clínica do recém-nascido durante sua internação.

Criou-se um modelo hierárquico com três níveis (Figura 1). O primeiro nível continha as variáveis pré-natais, tais como idade materna, categorizada em adolescentes (mães com idade $<20$ anos) e adultas (idade $\geq 20$ anos); ordem de nascimento (considerado primeiro ou segundo filho e, em outro grupo, ordem igual ou superior a terceiro filho); relato de natimorto anterior (ausente ou presente); número de consultas no pré-natal (seis ou menos e sete ou mais consultas); escolaridade materna (mães com até oito anos de escolaridade, ou seja, que completaram o primeiro grau, e mães com nove anos ou mais de escolaridade, com o segundo ou terceiro grau completo).

O segundo nível continha as variáveis perinatais: tipo de parto (vaginal ou cesáreo); peso do recém-nascido, considerando-se de baixo peso os bebês nascidos com peso <2500g; duração da gestação, considerando-se pré-termo os nascidos com idade gestacional <37 semanas; escore de Apgar no quinto minuto de vida, dividido em grupo com escore $<7$ e $\geq 7$.

O terceiro nível continha as variáveis pós-natais, que diziam respeito à evolução do recém-nascido durante a internação, levando-se em consideração procedimentos invasivos como ven- 
tilação mecânica (sim ou não), cateterização de vasos umbilicais (sim ou não), uso ou não de surfactante, relatos de episódios de convulsão ou apneia observados pela equipe médica.

Realizou-se análise bivariada entre óbito ou alta hospitalar e as variáveis de cada nível. Foram selecionadas, então, as variáveis que apresentaram nível de significância igual ou inferior a 0,20. A partir dessas variáveis, em cada nível e separadamente, realizou-se a análise multivariada, permanecendo no estudo aquelas que apresentaram nível de significância igual ou inferior a 0,05. Tais variáveis eram consideradas ajustadas pelas outras do mesmo nível.

$\mathrm{Na}$ segunda etapa, às variáveis do primeiro nível definidas, foram adicionadas as variáveis do segundo nível que apresentaram nível de significância igual ou menor que 0,20. Permaneceram nessa etapa do modelo as variáveis do segundo nível que mantiveram nível de significância igual ou inferior a 0,05 . Dessa forma, as variáveis do segundo nível estavam sendo ajustadas pelas variáveis do mesmo nível e do nível superior.

Da mesma forma, com o modelo contendo variáveis do primeiro e do segundo níveis, introduziram-se as variáveis do terceiro nível, as pós-natais. Como realizado na etapa anterior, mantiveram-se as variáveis do último nível cujo valor de $p$ fosse igual ou inferior a 0,05 . Construiu-se, portanto, um modelo hierárquico com variáveis dos três níveis que estimou os fatores de risco para óbito em UTIN.

Foram construídas tabelas com os valores das Odds Ratio da análise bivariada e multivariada, com os respectivos intervalos de confiança de 95\% (IC 95\%). Estimou-se a acurácia do modelo. Em algumas tabelas, os totais podem ser diferentes em virtude da falta de informação referente a alguma variável. As variáveis contínuas foram analisadas por meio do teste $t$ de Student. Para comparação de médias e regressão logística, utilizou-se a técnica stepwise forward com programas computacionais Epi-Info 6.04 e SPSS 10.0. A acurácia do modelo foi estimada pelo programa SPSS 10.0.

\section{Resultados}

Foram incluídos 367 recém-nascidos, sendo 212 (57,8\%) do sexo masculino e 155 (42,2\%) do feminino. O peso médio dos recém-nascidos incluídos foi $2132 \pm 824 \mathrm{~g}$. A idade gestacional média foi 35,1 $\pm 3,4$ semanas. O tempo de internação variou entre zero e 162 dias, com média de 18,9 dias. Ocorreram 69 óbitos, número correspondente a 18,8\% das internações.

Dos recém-nascidos, $252(68,7 \%)$ pertenciam ao grupo de baixo peso, $255(69,5 \%)$ eram prematuros e $113(30,8 \%)$ ti-

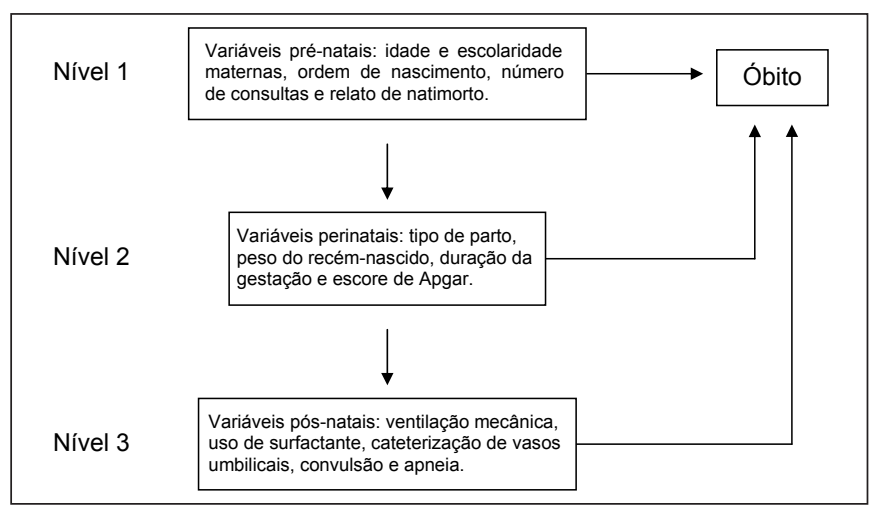

Figura 1 - Estrutura conceitual hierarquizada em blocos para óbito de RN em UTIN.

nham escore de Apgar no $5^{\circ}$ minuto de vida inferior a 7 . Havia $230(62,7 \%)$ pacientes com baixo peso e com idade gestacional inferior a 37 semanas (Tabela 1).

A Tabela 2 mostra a análise entre variáveis independentes do nível 1 e o desfecho em óbito, com as respectivas OR, intervalos de confiança de $95 \%$, valor de $p$ e o número de expostos com o respectivo percentual. Mantiveram-se, com significância $(p<0,20)$, as seguinte variáveis: idade materna, ordem de nascimento e relato de natimorto em gestação anterior. Excluíram-se as variáveis relativas à escolaridade materna e ao número de consultas no pré-natal.

A Tabela 3 mostra os valores obtidos pela análise entre variáveis independentes do nível 2 e a ocorrência do óbito. Com exceção do tipo de parto, que não apresentou significância estatística, as demais variáveis estiveram associadas ao óbito. O escore de Apgar de quinto minuto $\leq 7$ foi a variável que mais se associou à chance de óbito. Baixo peso ao nascer e prematuridade também foram fatores de risco para o óbito.

Das variáveis do período pós-natal, relatos de apneia ou de convulsão não se mostraram fatores de risco para o óbito. Já o uso de surfactante e a necessidade de cateterismo de vasos umbilicais e de ventilação mecânica se mostraram fatores de risco para óbito (Tabela 4).

O modelo final continha as variáveis: ordem de nascimento e relato de natimorto que ajustam as variáveis do segundo nível, Apgar no $5^{\circ}$ minuto de vida $<7$ e recém-nascido prétermo; a variável baixo peso perdeu a significância, sendo ajustada por essas duas variáveis do nível 2. Das variáveis do nível 3, só permaneceu a ventilação mecânica; as demais foram ajustadas entre elas e pelas variáveis dos níveis 1 e 2 , perdendo a significância estatística (Tabela 5).

A função logito obtida foi $\pi(\mathrm{x})=-4,08+2,17$ (Relato de natimorto) $+1,85$ (Primeiro ou segundo filho) $+3,84$ (pré-termo) $+8,89$ (Apgar de $5^{\circ}$ minuto $\left.<7\right)+5,71$ (Ventilação mecânica) . 
Tabela 1 - Análise bivariada segundo o tipo de saída (alta ou óbito) dos recém-nascidos internados na UTIN, com as porcentagens, valores de $p$ e número total de casos analisados (n) para cada variável independente e suas porcentagens em relação ao total de recém-nascidos

\begin{tabular}{|c|c|c|c|c|c|}
\hline & & Alta (\%) & Óbito (\%) & $p^{\#}$ & $n(\%) \S$ \\
\hline \multirow[t]{2}{*}{ Idade materna } & $<20$ anos & $73(26,6)$ & $11(18,6)$ & 0,20 & $333(9,3)$ \\
\hline & $\geq 20$ anos & $201(73,4)$ & $48(81,4)$ & & \\
\hline \multirow[t]{2}{*}{ Ordem de nascimento } & $1^{\circ}$ e $2^{\circ}$ filho & $139(46,6)$ & $40(57,9)$ & 0,09 & $367(0)$ \\
\hline & $3^{\circ}$ ou mais & $159(53,4)$ & $29(42,1)$ & & \\
\hline \multirow{2}{*}{ Relato prévio de natimorto } & Presente & $223(83,5)$ & $45(76,3)$ & 0,08 & $328(10,6)$ \\
\hline & Ausente & $44(16,5)$ & $16(23,7)$ & & \\
\hline \multirow[t]{2}{*}{ Consultas no pré-natal } & $<6$ & $224(75,2)$ & $53(76,8)$ & 0,79 & $367(0)$ \\
\hline & $\geq 7$ & $74(24,8)$ & $16(23,2)$ & & \\
\hline \multirow[t]{2}{*}{ Escolaridade materna } & Até 8 anos & $244(81,9)$ & $55(79,7)$ & 0,67 & $367(0)$ \\
\hline & $\geq 9$ anos & $54(18,1)$ & $14(20,3)$ & & \\
\hline \multirow{2}{*}{ Tipo de parto } & Vaginal & $148(49,8)$ & $38(55,9)$ & 0,37 & $365(0,5)$ \\
\hline & Cesáreo & $149(50,2)$ & $30(44,1)$ & & \\
\hline \multirow[t]{2}{*}{ Peso ao nascer } & Baixo & $196(65,8)$ & $56(81,2)$ & 0,013 & $367(0)$ \\
\hline & Normal & $102(34,2)$ & $13(18,8)$ & & \\
\hline \multirow[t]{2}{*}{ Gestação } & Termo & $93(31,8)$ & $59(85,5)$ & 0,004 & $361(1,6)$ \\
\hline & Pré-termo & $199(68,2)$ & $10(14,5)$ & & \\
\hline \multirow[t]{2}{*}{ Apgar 5 minutos } & $<7$ & $69(23,5)$ & $45(65,2)$ & $<0,001$ & $362(1,6)$ \\
\hline & $\geq 7$ & $224(76,5)$ & $24(34,8)$ & & \\
\hline \multirow[t]{2}{*}{ Ventilação mecânica } & Sim & $61(20,5)$ & $40(57,9)$ & $<0,001$ & $367(0)$ \\
\hline & Não & $237(79,5)$ & $29(42,1)$ & & \\
\hline \multirow[t]{2}{*}{ Cateterização } & Sim & $148(49,8)$ & $51(75,0)$ & 0,001 & $365(0,5)$ \\
\hline & Não & $149(50,2)$ & $17(25,0)$ & & \\
\hline \multirow[t]{2}{*}{ Uso de surfactante } & Sim & $30(10,1)$ & $23(33,3)$ & $<0,001$ & $367(0)$ \\
\hline & Não & $268(89,9)$ & $46(66,7)$ & & \\
\hline \multirow[t]{2}{*}{ Convulsão } & Sim & $11(3,7)$ & $4(5,9)$ & 0,42 & $346(5,7)$ \\
\hline & Não & $286(96,3)$ & $64(94,1)$ & & \\
\hline \multirow[t]{2}{*}{ Apneia } & Presente & $35(11,8)$ & $10(14,5)$ & 0,51 & $365(0,5)$ \\
\hline & Ausente & $262(88,2)$ & $58(85,5)$ & & \\
\hline
\end{tabular}

\# Obtido pelo teste do qui-quadrado; § Porcentagem de falta de informação em relação ao total de casos.

Tabela 2 - Análise bivariada entre variáveis do nível 1 e óbito, em recém-nascidos internados em UTIN, com as respectivas Odds Ratio brutas (OR), intervalos de confiança de 95\% (IC 95\%), p e número e percentual de expostos

\begin{tabular}{lcccc}
\hline & OR & IC 95\% & $\boldsymbol{p}$ & Expostos (\%) \\
\hline Idade materna menor que 20 anos & 1,57 & $0,73-3,42$ & 0,20 & $11(13,1)$ \\
Escolaridade materna $\leq 8$ anos & 1,00 & $0,49-2,02$ & 0,67 & $14(24,1)$ \\
Consultas no pré-natal $\leq 6$ & 1,14 & $0,57-2,32$ & 0,79 & $16(17,8)$ \\
Relato de natimorto & 1,77 & $0,87-3,59$ & 0,08 & $45(16,8)$ \\
Primeiro ou segundo filho & 1,65 & $0,89-3,03$ & 0,09 & $40(22,2)$ \\
\hline
\end{tabular}

Tabela 3 - Análise bivariada entre variáveis do nível 2 e óbito, em recém-nascidos internados em UTIN, com as respectivas Odds Ratio brutas (OR), intervalos de confiança de 95\% (IC 95\%), p e número e percentual de expostos

\begin{tabular}{lcccc}
\hline & OR & IC 95\% & $\boldsymbol{p}$ & Expostos (\%) \\
\hline Baixo peso ao nascer & 2,27 & $1,12-4,54$ & 0,01 & $56(22,2)$ \\
Pré-termo & 2,94 & $1,33-6,66$ & 0,003 & $59(38,8)$ \\
Apgar $<7$ & 5,88 & $3,22-11,11$ & $<0,001$ & $45(39,5)$ \\
Parto cesáreo & 0,78 & $0,44-1,38$ & 0,37 & $38(20,4)$ \\
\hline
\end{tabular}


Tabela 4 - Análise bivariada entre variáveis do nível 3 (variáveis pós-natais) e óbito, em recém-nascidos internados em UTIN com as respectivas Odds Ratio brutas (OR), intervalos de confiança de 95\% (IC 95\%), p e número e percentual de expostos

\begin{tabular}{lcccc}
\hline & OR & IC 95\% & $\boldsymbol{p}$ & Expostos (\%) \\
\hline Uso de surfactante & 4,52 & $2,29-8,91$ & $<0,001$ & $23(43,4)$ \\
Cateterismo & 2,96 & $1,57-5,64$ & $<0,001$ & $51(25,6)$ \\
Apneia & 1,29 & $0,56-2,91$ & 0,52 & $10(22,2)$ \\
Convulsão & 1,62 & $0,42-5,82$ & 0,41 & $4(26,7)$ \\
Ventilação mecânica & 5,83 & $3,16-10,70$ & $<0,001$ & $40(65,6)$ \\
\hline
\end{tabular}

Tabela 5 - Variáveis do modelo final e respectivos Odds Ratio (OR) ajustadas, intervalos de confiança de 95\% (IC 95\%) e valores de $p$

\begin{tabular}{lccc}
\hline & OR & IC 95\% & p \\
\hline Relato de natimorto & 2,17 & $1,09-4,30$ & $0,03^{\#}$ \\
Primeiro ou segundo filho & 1,85 & $1,02-3,33$ & $0,04^{\#}$ \\
Pré-termo & 3,84 & $1,65-8,90$ & $0,002^{*}$ \\
Apgar $<7$ & 8,89 & $4,56-17,36$ & $<0,001^{*}$ \\
Ventilação mecânica & 5,71 & $2,83-11,50$ & $<0,001^{* *}$ \\
\hline
\end{tabular}

Hosmer-Lemeshow goodness-of-fit qui-quadrado=7,5 ( $p=0,38)$; Constante $\beta_{0}=-4,08$; \# Após ajuste com as variáveis do nível 1 ; *Após ajuste com as variáveis do mesmo nível e do nível $1 ;{ }^{* *}$ Após ajuste com as variáveis do mesmo nível e dos níveis 1 e 2 .

No caso de um recém-nascido que apresentasse todas as variáveis acima, a chance de óbito seria de quase $100 \%$; por outro lado, um recém-nascido prematuro sem as demais características desse grupo apresentaria risco de óbito de aproximadamente $44 \%$. Esse modelo mostrou uma acurácia de 86,9\%.

\section{Discussão}

O objetivo deste estudo foi estimar os fatores de risco para a mortalidade em uma Unidade de Terapia Intensiva Neonatal de um hospital universitário. Esta análise incluiu fatores ocorridos durante a internação hospitalar, isto é, relativos a procedimentos médicos realizados nos recém-nascidos, e fatores ligados aos períodos pré-natal e perinatal. A UTIN onde foi realizado este estudo pertence a um hospital universitário que atende principalmente pacientes nascidos no próprio hospital, mas também provenientes de municípios da região, com predominância de usuários do Sistema Único de Saúde.

A taxa de mortalidade foi inferior à encontrada em outros estudos ${ }^{(5,12-14)}$ e superior aos achados de Zullini et al ${ }^{(11)}$ e Araújo et al ${ }^{(15)}$. Possivelmente, essas diferenças tenham ocorrido em virtude da época do estudo ou das características da população atendida.

Quanto aos determinantes distais, representados pelas variáveis do primeiro nível, relatos de natimorto em outra gestação e ordem de nascimento, estas se associaram ao óbito durante a internação. Associação entre mortalidade neonatal e relato prévio de natimorto foi constatada em um estudo realizado em São Paulo, em 1995, com dados obtidos do SIM e Sinasc, não havendo relato específico de óbitos em UTIN. Talvez essa associação se deva a um pré-natal feito sem a atenção necessária ou a fatores maternos que não permitiram levar a gravidez a bom termo. A ordem de nascimento foi apontada por Weirich et al ${ }^{(5)}$ e por Duarte e Mendonça ${ }^{(16)}$ como importante fator relacionado ao óbito de recém-nascidos.

Dentre as variáveis do segundo nível, o tipo de parto não mostrou associação significante com o óbito tanto na análise bivariada como na multivariada, o que está de acordo com outros estudos ${ }^{(3,4)}$. Por outro lado, o escore de Apgar no $5^{\circ}$ minuto de vida, que indica baixa vitalidade, o baixo peso ao nascer e a prematuridade foram identificados como fatores de risco para a mortalidade neonatal em outros estudos ${ }^{(3-5,17)}$. Na análise bivariada da presente pesquisa, esses também foram fatores de risco para o óbito, mas na análise multivariada o baixo peso ao nascer perdeu a significância, sendo ajustado pela prematuridade, o que coincide com os achados de Barros et al ${ }^{(18)}$. Possivelmente, um resultado diferente seria obtido se essa variável incluísse um grupo com peso inferior a $1500 \mathrm{~g}$.

Durante a internação, várias medidas são tomadas para corrigir intercorrências graves do recém-nascido internado. $\mathrm{O}$ cateterismo de vasos umbilicais costuma ser quase uma 
rotina, com o objetivo de estabelecer uma via de fácil acesso para infusão de líquidos e medicamentos e para a coleta de sangue para exames laboratoriais. Como os estudos sobre mortalidade neonatal geralmente se restringem a fatores determinados pelas informações de registros oficiais, pouco se sabe do papel das medidas de manipulação no óbito do recém-nascido internado. No presente estudo, essa variável teve significância estatística tanto na análise bivariada como na multivariada, no mesmo nível. Quando incluída no modelo final, foi ajustada pelas outras dos níveis anteriores e perdeu sua significância. $\mathrm{O}$ mesmo aconteceu com o uso de surfactante, cuja indicação consiste na prevenção/tratamento da doença das membranas hialinas. Sua inclusão no modelo final representou perda de sua significância estatística por ajuste pelas outras variáveis, principalmente pela ventilação mecânica. Essa situação foi semelhante à encontrada por Duarte e Mendonça ${ }^{(16)}$, que observaram a importância desse fator na análise univariada, mas houve desaparecimento de sua significância na análise multivariada. Talvez a explicação esteja, segundo tais autores, no uso não adequado dessa medida terapêutica ${ }^{(16)}$. Outras duas variáveis, apneia e convulsão, não se associaram de forma significante ao óbito intra-hospitalar na análise bivariada nem na multivariada.

Restou a ventilação mecânica como fator de risco para o óbito durante a internação, mostrando-se fortemente associada ao óbito de modo isolado e em conjunto, no modelo final de regressão logística. Assim, pode-se estimar que o fato de o recém-nascido estar em ventilação mecânica aumenta a chance de óbito em quase seis vezes. Mesmo após ajuste por todas as outras variáveis, a ventilação mecânica continuou fortemente associada ao óbito. $\mathrm{O}$ fato de o neonato necessitar de ventilação mecânica sugere gravidade clínica, e vem daí sua importância para o óbito durante a internação hospitalar em uma UTIN. A ventilação mecânica foi identificada também como fator de risco para o óbito em outro estudo ${ }^{(16)}$.

O modelo aqui apresentado tem limitações. Dentre elas, podem ser apontadas: falta de informação sobre problemas clínicos maternos como hipertensão arterial, diabetes e sangramento durante a gestação; falta de informações sobre uso de corticoide no pré-natal; além de ausência de dados da evolução clínica do neonato durante a internação, como o diagnóstico de certeza de sepse neonatal e o uso de nutrição parenteral que, em outros estudos, associam-se ao óbito ${ }^{(15,16)}$. Pode-se citar, ainda, a ausência da informação sobre a procedência do recém-nascido, pois sendo de outro município, mesmo que o transporte seja realizado por serviço especializado, essa variável pode ter um papel importante no óbito.

Quanto aos diagnósticos associados ao óbito, neste estudo foram encontrados os seguintes: sepse, hemorragia peri e intraventricular, doença das membranas hialinas, enterocolite necrosante e outras afecções do aparelho respiratório (dados não mostrados). Com uma casuística maior, alguns desses diagnósticos poderão se mostrar, a partir da regressão logística, fatores de risco mais ou menos importantes para o óbito em UTIN.

O modelo proposto mostrou um bom ajuste, evidenciado pelo resultado do teste de Hosmer-Lemeshow. As variáveis que apresentaram maior peso para o óbito nesta amostra foram prematuridade, baixa vitalidade (Apgar no quinto minuto $<7)$ e ventilação mecânica. O recém-nascido prétermo, isoladamente, apresentou uma chance de óbito em torno de $44 \%$ e aquele portador de todas as variáveis do modelo mostrou quase $100 \%$ de chance de óbito. O modelo obtido evidenciou, também, acurácia considerável $(86,9 \%)$. Esse modelo pode ser utilizado em outras UTIN que tenham características semelhantes às do hospital universitário aqui analisado.

\section{Referências bibliográficas}

1. Hoyert DL, Freedman MA, Strobino DM, Guyer B. Annual summary of vital statistics: 2000. Pediatrics 2001;108:1241-55.

2. Sarinho SW, Filho DA, Silva GA, Lima MC. Risk factors for neonatal death in Recife: a case-control study. J Pediatr (Rio J) 2001;77:294-8.

3. Helena ET, Sousa CA, Silva CA. Risk factors for neonatal mortality in Blumenau, Santa Catarina: linkage between database. Rev Bras Saude Matern Infant 2005;5:209-17.

4. Martins EF, Velásquez-Meléndez G. Determinants of neonatal mortality in a cohort of born alive infants, Montes Claros, Minas Gerais, 1997-1999. Rev Bras Saude Matern Infant 2004;4:405-12.
5. Weirich CF, Andrade AL, Turchi MD, Silva SA, Morais-Neto OL, Minamisava $R$ et al. Neonatal mortality in intensive care unit of Central Brazil. Rev Saude Publica 2005;39:775-81.

6. Mendes KG, Olinto MT, Costa JS. Case-control study on infant mortality in southern Brazil. Rev Saude Publica 2006;40:240-8.

7. Pollack MM, Ruttimann UE, Getson PR. Accurate prediction of the outcome of pediatric intensive care. A new quantitative method. N Engl J Med 1987;316:134-9.

8. Richardson DK, Gray JE, McCormick MC, Workman K, Goldmann DA. Score for neonatal acute physiology: physiologic severity index for neonatal intensive care. Pediatrics 1993;91:617-23. 
9. No authors listed. The CRIB (critical risk index for babies) score: a tool assessing initial neonatal risk and comparing performance of neonatal intensive care units. International Neonatal Network. Lancet 1993;342:193-8.

10. Nascimento LF, Ortega NR. Fuzzy linguistic model for evaluating the risk of neonatal death. Rev Saude Publica 2002;36:686-92.

11. Zullini MT, Bonati M, Sanvito E. Survival at nine neonatal intensive care units in São Paulo, Brazil. Paulista Collaborative Group on Neonatal Care. Rev Panam Salud Publica 1997;2:303-9.

12. Sarquis AL, Miyaki M, Cat MN. The use of CRIB score for predicting neonatal mortality risk. J Pediatr (Rio J) 2002;78:225-9.

13. Nascimento LF, Ramos RS. The use of CRIB score as mortality predictor at neonatal intensive care unit: an extended approach. Rev Bras Saude Matern Infant 2004;4:151-7.

14. Matsuoka OT, Sadeck LS, Haber JF, Proença RS, Mataloun MM, Ramos JL et al. Predictive value of the "Clinical Risk Index for Ba- bies" for the risk of neonatal death. Rev Saude Publica 1998;32: 550-5.

15. Araújo BF, Tanaka AC, Madi JM, Zatti H. Newborn mortality study in the neonatal intensive care unit of Caxias do Sul General Hospital, Rio Grande do Sul. Rev Bras Saude Matern Infant 2005;5:463-9.

16. Duarte JL, Mendonça GA. Factors associated with neonatal mortality among very low birthweight newborns in four maternity hospitals in the city of Rio de Janeiro, Brazil. Cad Saude Publica 2005;21:181-91.

17. Morais-Neto OL, Barros MB. Risk factors for neonatal and post-neonatal mortality in the Central-West region of Brazil: linkage between live birth and infant death data banks. Cad Saude Publica 2000;16:477-85.

18. Barros FC, Victora CG, Barros AJ, Santos IS, Albernaz E, Matijasevich A et al. The challenge of reducing neonatal mortality in middle-income countries: findings from three Brazilian birth cohorts in 1982, 1993, and 2004. Lancet 2005;365:847-54. 